\title{
Correction to: Maternal personality disorder symptoms in primary health care: associations with mother-toddler interactions at one-year follow-up
}

Magnhild Singstad Høivik ${ }^{1,3^{*}}$, Stian Lydersen², Ingunn Ranøyen ${ }^{2}$ and Turid Suzanne Berg-Nielsen ${ }^{4}$

\section{Correction to: BMC Psychiatry (2018) 18:198}

\section{DOI: 10.1186/s12888-018-1789-5}

Following publication of the original article [1], the authors notified us of an error in the data presented in Table 2: the DIP-Q subscales show an incorrect scale.

The corrected Table 2 is presented below.

\begin{abstract}
Author details
'Department of Mental Health, Faculty of Medicine and Health Sciences, the Norwegian University of Science and Technology (NTNU), N-7491 Trondheim, Norway. ${ }^{2}$ Regional Centre for Child and Youth Mental Health and Child Welfare - Central Norway, Faculty of Medicine and Health Sciences, The Norwegian University of Science and Technology (NTNU), Trondheim, Norway. ${ }^{3}$ Division of Psychiatry, St Olavs Hospital, Trondheim University Hospital, Trondheim, Norway. ${ }^{4}$ The Centre for Child and Adolescent Mental Health, Eastern and Southern Norway, Oslo, Norway.
\end{abstract}

Received: 23 November 2018 Accepted: 23 November 2018 Published online: 13 December 2018

\section{Reference}

1. Høivik, et al. Maternal personality disorder symptoms in primary health care: associations with mother-toddler interactions at one-year follow-up. 2018; 18:198. https://doi.org/10.1186/s12888-018-1789-5.

\footnotetext{
* Correspondence: magnhild.s.hoivik@ntnu.no

${ }^{1}$ Department of Mental Health, Faculty of Medicine and Health Sciences, the Norwegian University of Science and Technology (NTNU), N-7491 Trondheim, Norway

${ }^{3}$ Division of Psychiatry, St Olavs Hospital, Trondheim University Hospital,

Trondheim, Norway

Full list of author information is available at the end of the article
}

(c) The Author(s). 2018 Open Access This article is distributed under the terms of the Creative Commons Attribution 4.0 International License (http://creativecommons.org/licenses/by/4.0/), which permits unrestricted use, distribution, and reproduction in any medium, provided you give appropriate credit to the original author(s) and the source, provide a link to the Creative Commons license, and indicate if changes were made. The Creative Commons Public Domain Dedication waiver (http://creativecommons.org/publicdomain/zero/1.0/) applies to the data made available in this article, unless otherwise stated. 
Table 2 Sample clinical characteristics at baseline and at one-year follow-up

\begin{tabular}{|c|c|c|c|c|c|c|c|c|c|c|c|c|c|c|c|}
\hline \multicolumn{10}{|l|}{ Baseline } & \multicolumn{6}{|c|}{ Follow up } \\
\hline \multirow[b]{2}{*}{$\begin{array}{l}\text { Personality disorder } \\
\text { symptoms }\end{array}$} & \multicolumn{3}{|c|}{ All participants } & \multicolumn{3}{|c|}{ Attrition group } & \multicolumn{3}{|c|}{ Remaining group } & \multirow[b]{2}{*}{$n$} & \multirow[b]{2}{*}{ mean } & \multirow[b]{2}{*}{$\mathrm{sd}$} & \multirow[b]{2}{*}{$\begin{array}{l}\text { cut off } \\
\text { (diagnosis) }\end{array}$} & \multirow[b]{2}{*}{$\begin{array}{l}\text { n (\%) with symptoms } \\
\text { over cut off }\end{array}$} & \multirow[b]{2}{*}{$\begin{array}{l}\text { Scale } \\
\text { range }\end{array}$} \\
\hline & $\mathrm{n}$ & mean & $\mathrm{sd}$ & $\mathrm{n}$ & mean & $s d$ & $\mathrm{n}$ & mean & sd & & & & & & \\
\hline Avoidant & 122 & 1.93 & 1.95 & 28 & 2.29 & 2.56 & 94 & 1.82 & 1.85 & - & - & - & $\geq 4$ & $26(21.3)$ & $0-7$ \\
\hline Dependent & 122 & 1.81 & 1.88 & 28 & $2.61^{* * *}$ & 2.39 & 94 & $1.57^{* * *}$ & 1.64 & - & - & - & $\geq 5$ & $13(10.7)$ & $0-9$ \\
\hline Obsessive-compulsive & 122 & 3.88 & 1.74 & 28 & 4.00 & 1.89 & 94 & 3.84 & 1.70 & - & - & - & $\geq 4$ & 69 (56.6) & $0-9$ \\
\hline Paranoid & 122 & 1.34 & 1.60 & 28 & $2.04^{* * *}$ & 2.03 & 94 & $1.14^{* * * *}$ & 1.40 & - & - & - & $\geq 4$ & $8(6.6)$ & $0-8$ \\
\hline Schizoid & 122 & 0.73 & 0.97 & 28 & 0.68 & 0.86 & 94 & 0.75 & 1.00 & - & - & - & $\geq 4$ & $1(0.8)$ & $0-8$ \\
\hline Schizotypal & 122 & 0.41 & 1.66 & 28 & 2.04 & 1.88 & 94 & 1.22 & 1.55 & - & - & - & $\geq 5$ & $10(8.2)$ & $0-11$ \\
\hline Antisocial & 122 & 0.85 & 0.85 & 28 & 0.86 & 0.85 & 94 & 0.85 & 0.94 & - & - & - & $\geq 3$ & $6(4.9)$ & $0-10$ \\
\hline Borderline & 122 & 2.48 & 2.15 & 28 & $3.61^{* * *}$ & 2.69 & 94 & $2.14^{* * *}$ & 1.85 & - & - & - & $\geq 5$ & 19 (15.6) & $0-17$ \\
\hline Histrionic & 122 & 1.25 & 1.22 & 28 & 1.39 & 1.17 & 94 & 1.21 & 1.24 & - & - & - & $\geq 5$ & $1(0.8)$ & $0-8$ \\
\hline Narcissistic & 122 & 0.86 & 1.05 & 28 & $1.29^{* *}$ & 1.33 & 94 & $0.73^{* *}$ & 0.87 & - & - & - & $\geq 5$ & $1(0.8)$ & $0-10$ \\
\hline Impairment and distress & 122 & 0.66 & 1.03 & 28 & $0.80^{*}$ & 1.26 & 94 & $0.63^{*}$ & 0.96 & - & - & - & $\geq 2$ & $20(16.4)$ & $0-5$ \\
\hline Depressive symptoms & 118 & 12.11 & 8.64 & 24 & $15.96^{*}$ & 11.23 & 94 & $10.99^{* *}$ & 7.84 & 85 & $8.74^{* * *}$ & 7.05 & - & - & $0-63$ \\
\hline \multicolumn{16}{|l|}{ EAS subscales } \\
\hline Maternal sensitivity & 152 & 22.41 & 5.12 & 42 & 21.48 & 4.94 & 110 & 22.77 & 5.16 & 110 & $25.29^{* * *}$ & 3.92 & - & - & $7-29$ \\
\hline Maternal structuring & 152 & 23.26 & 4.50 & 42 & 22.38 & 4.29 & 110 & 23.60 & 4.55 & 110 & $25.90^{* * *}$ & 3.39 & - & - & $7-29$ \\
\hline Maternal non-hostility & 152 & 26.01 & 3.58 & 42 & $24.92^{*}$ & 4.18 & 110 & $26.44^{*}$ & 3.24 & 110 & $27.32^{* * *}$ & 2.50 & - & - & $7-29$ \\
\hline $\begin{array}{l}\text { Maternal } \\
\text { non-intrusiveness }\end{array}$ & 152 & 22.24 & 5.72 & 42 & 21.26 & 6.16 & 110 & 22.63 & 5.52 & 110 & $25.27^{* * *}$ & 4.34 & - & - & $7-29$ \\
\hline Child responsiveness & 152 & 22.66 & 5.36 & 42 & 21.59 & 5.06 & 110 & 23.07 & 5.43 & 110 & $25.70^{* * *}$ & 4.08 & - & - & $7-29$ \\
\hline Child involvement & 152 & 22.43 & 5.94 & 42 & 20.50 & 5.97 & 110 & 21.79 & 5.92 & 110 & $25.11^{* * *}$ & 4.71 & - & - & $7-29$ \\
\hline
\end{tabular}

Maternal personality disorder symptoms measured with the DIP-Q (DSM IV and ICD-10 Personality Questionnaire)

${ }^{*}=p<0.05,{ }^{* *}=p<0.01,{ }^{* * *}=p<0.001$ (independent samples t-tests of characteristics in attrition compared to remaining groups in baseline sample, and paired sample t-tests comparing characteristics of remaining and follow up groups) 